\title{
P 160 KNOWLEDGE AND PRACTICE OF PALLIATIVE CARE PROFESSIONALS IN PREVENTION OF PATHOLOGICAL FRACTURE
}

Maria Debattista, ${ }^{1}$ Andrew F Khodabukus, ${ }^{2}$ Joanne Reynolds, ${ }^{3}$ Paula Horton, ${ }^{4}$ Susan Howarth, ${ }^{4}$ Barbara Humphries, ${ }^{5}$ Jennifer C Smith ${ }^{5} .{ }^{1}$ Marie Curie Hospice Liverpool, Liverpool, United Kingdom; ${ }^{2}$ Marie Curie Palliative Care Institute Liverpool, United Kingdom; ${ }^{3}$ The Clatterbridge Cancer Centre NHS Foundation Trust, Wirral, United Kingdom; ${ }^{4}$ Royal Liverpool and Broadgreen University Hospital NHS Trust, Liverpool, United Kingdom; ${ }^{5}$ Countess of Chester NHS Foundation Trust, Chester, United Kingdom

\subsection{6/bmjspcare-2014-000654.201}

Background Metastatic bone disease (MBD) has a damaging effect on quality of life and pathological fracture can impact on survival in advanced cancer. Patients with breast and prostatic cancer in particular who have bone metastases have a median life expectancy in excess of two years. It is essential that there is identification of patients who may benefit from surgical management that can prevent pathological fracture and its associated morbidity. There is recognition that there is under-referral to orthopaedic surgeons for consideration of palliative surgery and in 2005 guidelines were produced to support clinicians.

Aims To identify the preferences and actions of doctors and clinical nurse specialists in assessing bone pain in adult patients with advanced cancer compared with the 2005 regional standards and guidelines.

Methods Two simultaneous surveys were done evaluating selfassessed clinical practice and a retrospective case-note review of assessments made by specialist palliative care teams. These were disseminated across integrated palliative care clinical networks in the North West of England.

Results 38 evaluations of self-assessed clinical practice were completed. 29\% were unaware of guidance to support evaluation of MBD and pathological fracture. $66 \%$ of participants were unaware of their local lead orthopaedic surgeon for MBD. 69 case note reviews were completed. In the 44 patients not know to have metastatic bone disease at the site of pain $57 \%$ did not have an assessment of risk of metastatic disease in keeping with standards. 35 had assessments where risk of pathological fracture could be graded and in $80 \%$ of these no risk was documented.

Conclusions Despite the high frequency of bone pain there is insufficient documentation of risk of MBD and risk of pathological fracture. The results present an opportunity for clinicians to discuss local management options within palliative care services and with orthopaedic surgeons and oncologists. 